2. P. J. Cohen, Factorization in group algebras, Duke Math. J. 26 (1959), 199-205.

3. N. Jacobson, Structure of rings, Colloq. Publ., Vol. 37, Amer. Math. Soc., Providence, R. I., 1956.

4. I. Kaplansky, Dual rings, Ann. of Math. 49 (1948), 689-701.

5. J. D. Newburgh, The variation of spectra, Duke Math. J. 18 (1951), 165-176.

6. C. E. Rickart, Banach algebras, Van Nostrand, Princeton, N. J., 1960.

7. B. Yood, Homomorphisms on normed algebras, Pacific J. Math. 8 (1958), 373381.

The University of California, Berkeley and

The UNIVERSITY OF Oregon

\title{
GROUPS AND SEMIGROUPS WITH SOLVABLE WORD PROBLEMS
}

B. H. MAYOH

This note gives a direct proof of the following theorem of M. O. Rabin [1, Theorem 4]:

THEOREM. A finitely generated group has a solvable word problem (with respect to a given system of generators) if and only if it is computable.

A group is said [1] to have a solvable word problem with respect to a finite set of generators $\left\{g_{1}, \cdots, g_{n}\right\}$ if one can effectively determine whether or not two words on $\left\{\sigma_{1}, \cdots, \sigma_{n}\right\}$ represent the same group element. (Rabin's definition, though equivalent for groups, prevents the theorem from applying to semigroups. For convenience we suppose that the inverse of a generator is a generator.) A group is said to be computable if it has a recursive realization $\{S, x\}$-i.e. it is isomorphic to the group formed by a recursive subset $S$ of the positive integers and a recursive function $x(i, j)$ on $S$ that satisfies the group multiplication axioms.

Proof. Consider some recursive realization, $\{S, x\}$ of a given finitely generated computable group. Let $s_{i}$ be the element of $S$ corresponding to the generator $g_{i}$. For any word $W=\sigma_{a} \sigma_{b} \cdots \sigma_{z}$ one can effectively compute the in teger

$$
w=s_{a} \times s_{b} \times \cdots \times s_{z} .
$$

As two such words, $W$ and $W^{1}$, are equivalent if and only if $w=w^{1}$,

Received by the editors September 15, 1966. 
our group has a solvable word problem with respect to the given set of generators. Conversely suppose that a group has a solvable word problem with respect to some finite set of generators $\left\{g_{1}, \cdots, g_{m}\right\}$. One can effectively list the words on $\left\{\sigma_{1}, \cdots, \sigma_{m}\right\}$ lexicographically; since the word problem is solvable one can even do this omitting any word that represents the same group element as some word already listed: $W_{1}, W_{2}, W_{3}, \cdots$. Let $S$ be the set of indices in this list (it is either the positive integer or a finite segment thereof). For any $i$ and $j$ define $x(i, j)$ as:

$(\mu k)\left\{W_{k}\right.$ represents the same group element as $\left.W_{i} W_{j}\right\}$.

Clearly $\{S, x\}$ is a recursive realization of our group.

REMARK 1. One can replace "group" by "semigroup" throughout the above theorem and its proof.

REMARK 2. As noted in [1], our theorem implies that "having a solvable word problem" is a property of the group and not of the manner in which it is presented.

\section{Reference}

1. M. O. Rabin, Computable algebra, general theory and theory of computable fields, Trans. Amer. Math. Soc. 95 (1960), 341-360.

UNIVERSITY OF AARHUS 